\title{
A robo-pigeon based on an innovative multi- mode telestimulation system
}

\author{
Junqing Yang ${ }^{\mathrm{a}, \mathrm{b},{ }^{*}}$, Ruituo Huai ${ }^{\mathrm{b}}$, Hui Wang ${ }^{\mathrm{b}}$, Changzhi Lv ${ }^{\mathrm{b}}$ and Xuecheng $\mathrm{Su}^{\mathrm{b}}$ \\ ${ }^{a}$ Institute of RF and OE-ICs, Southeast University, Nanjing 210096, China \\ ${ }^{b}$ College of Electrical Engineering and Automation, Shandong University of Science and Technology, \\ Qingdao Shandong Province, 266590, China
}

\begin{abstract}
In this paper, we describe a new multi-mode telestimulation system for brain-microstimulation for the navigation of a robo-pigeon, a new type of bio-robot based on Brain-Computer Interface (BCI) techniques. The multi-mode telestimulation system overcomes neuron adaptation that was a key shortcoming of the previous single-mode stimulation by the use of non-steady TTL biphasic pulses accomplished by randomly alternating pulse modes. To improve efficiency, a new behavior model ("virtual fear") is proposed and applied to the robo-pigeon. Unlike the previous "virtual reward" model, the "virtual fear" behavior model does not require special training. The performance and effectiveness of the system to alleviate the adaptation of neurons was verified by a robo-pigeon navigation test, simultaneously confirming the practicality of the "virtual fear" behavioral model.
\end{abstract}

Keywords: Robo-pigeon, bio-robot, brain-computer interface, telestimulation system, multi-mode

\section{Introduction}

Brain computer interface (BCI) research began in the 1970s, and can be segmented into the categories of invasive BCIs, partially invasive BCIs and non-invasive BCIs. Invasive BCI research has targeted the repair of damaged sight, hearing and movement to provide new functionality for people experiencing paralysis. Partially invasive BCIs have been used to measure the electrical activity of the brain or a single neuron with high spatial resolution and low noise. Non-invasive BCI research has been primarily focused on electroencephalography (EEG) and has generally targeted brain-control of prosthetic devices. BCI technology creates a conduit for direct communication between the brain and a computer by means of electrodes. Over the course of just a few decades, this technology has opened up a range of clinical applications, such as treatments for Parkinson's disease and profound deafness. In particular, it is possible to utilize BCI technology in the development of robotics, leading to the development of a new category of robot referred to as robo-animals. Robo-animals differ from traditional robots in structure and principle. They can navigate obstacles better than electromechanical robots because they have superior physical strength and flexibility. Moreover, robo-animals incorporate an animal's visual, audio and tactile sensory capabilities, which make the robo-animals

\footnotetext{
* Address for correspondence: Junqing Yang, Institute of RF and OE-ICs, Southeast University, Jiangsu Province, 210096, China. Tel.: +086-25-83793303; Fax:+086-25-83792882; E-mail: yjqings2005@163.com.
} 
more intelligent without the requirement of any extraneous attachments.

Robo-animals could be utilized as couriers to locations not easily accessible to humans or mechanical robots. For example, robo-animals could be equipped with sensors to detect survivors trapped under the rubble caused by an earthquake. The ability to control an animal's behavior would also be useful in studies of animal communication and mating behavior. The many potential applications of robo-animals has expanded the focus of biomedical engineering to include the development of robust telemetry systems [1-3], stimulation systems [4-7] and behavior models specifically designed for robo-animals.

Recent research has demonstrated that BCI technology can be used to control an animal's actions and behavior through the use of micro-electrical stimulation. Shimoyama has described a robo-roach that can be made to move forward and turn left or right by stimulating the insect's nerves [8]. In 2002, the first robo-rat was developed based on a virtual reward system [9]. Then, flight control of a cyborg beetle was achieved via neural and muscular stimulation [10, 11]. Despite major advances [12-17], robo-animals cannot travel far and continue to display poor performance due to some common difficulties. For example, the stimulators in robo-animals are occasionally too large to successfully implant into an animal, the communication distance between the stimulator and remote control is limited, and the control of bio-robot navigation is unstable because of the adaptation of animal neurons. This neuron adaptation occurs when the discharge frequency of the neurons is reduced due to prolonged steady electrical stimulation [18-20]. This problem may be alleviated by a new multi-mode telestimulation system for brain-microstimulation, which overcomes some of the shortcomings of previous remote stimulators by outputting multi-mode and non-steady electrical stimuli.

\section{Methods}

A multi-mode telestimulation system and a new behavior model of the robo-animal are described in the context of robo-pigeon navigation experiments. The multi-mode telestimulation system enables an experimenter to remotely deliver multi-mode electrical stimuli to various sites in the brain of a freely moving pigeon. The behavior model of the robo-pigeon is based on virtual fear, which differs from the virtual reward system employed in the robo-rat experiments [9]. The primary feature of this behavioral model is that it does not require special training, as is required by the virtual reward model.

\subsection{Behavior model of the robo-bird}

Three mood-related brain sites were selected as target locations for micro-electrical stimulation based on virtual fear: the left and right nucleus dorsalis intermedius ventralis anterior (DIVA), and the periaqueductal gray $(\mathrm{PAG})$ region. Six resin-coated stainless steel electrodes (tip diameter $=30 \mu \mathrm{m}$ ) were paired off, and two electrodes of each pair were deposed, spaced $1 \mathrm{~mm}$ apart. The three paired electrodes were surgically implanted stereotaxically into the three brain sites. Stimulation experiments commenced after surgical rehabilitation (approximately seven days). The robo-pigeon turned left or right to escape the virtual fear from the right or left side of the body when the operator stimulated the left or right DIVA, respectively. Similarly, the robo- pigeon was moved forward by stimulating the PAG.

\subsection{Multi-mode telestimulation system}


The multi-mode telestimulation system consisted of two main components: a micro-embedded computer with a transmitter, and an integrated receiver-microprocessor module mounted on the pigeon's head and connected to the implanted electrodes, as shown in Figure 1. A program running on the micro-embedded computer provided the operator with a user interface to set and adjust the stimulation parameters. Digital commands were sent remotely to the receiver-microprocessor module by clicking the buttons on the user interface. The receiver-microprocessor module executed the incoming commands and delivered biphasic TTL pulses to the specified brain region.

\subsubsection{Micro-embedded computer with transmitter}

The micro-embedded computer was a typical embedded system in that it consisted of hardware based on the MPU S3C6410 (ARM11 core, Samsung Inc.) system software that is in turn based on the WinCE6.0 (Microsoft) embedded operating system. The user software was written in $\mathrm{C}++$ using Visual Studio 2005 (Microsoft).

The transmitter was a unit module based on the RF chip nRF401 (Nordic Inc.), which was connected to the S3C6410 by a serial port. The parameters and commands were sent as an ASCII string, at 9600 baud, from the micro-embedded computer to the transmitter via the serial port. The parameters included pulse frequency, pulse duration, and number of pulses.

\subsubsection{Multi-mode receiver-microprocessor}

The previously described receiver-microprocessor generated only a single-mode pulse train. This resulted in poor performance whenever continuous navigation of the robo-pigeon was required because the neurons adapted to the stimulus and eventually ceased to respond. We designed a new receiver-microprocessor to output a multi-mode pulse train to alleviate this neuronal adaptation. Figure 2 shows the three ideal waveforms of the multi-mode pulse trains.

A special feature of the receiver-microprocessor was its output of non-steady and multi-mode stimuli by randomly alternating pulse modes. The receiver-microprocessor was primarily comprised of

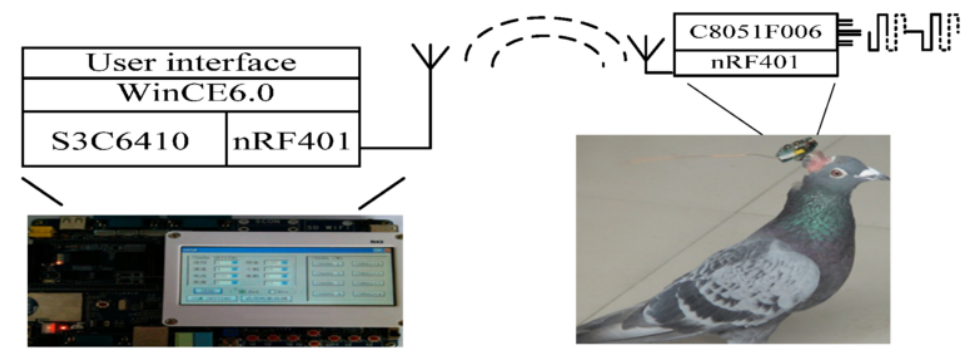

Fig. 1. Overview of robo-pigeon and multi-mode telestimulation system.
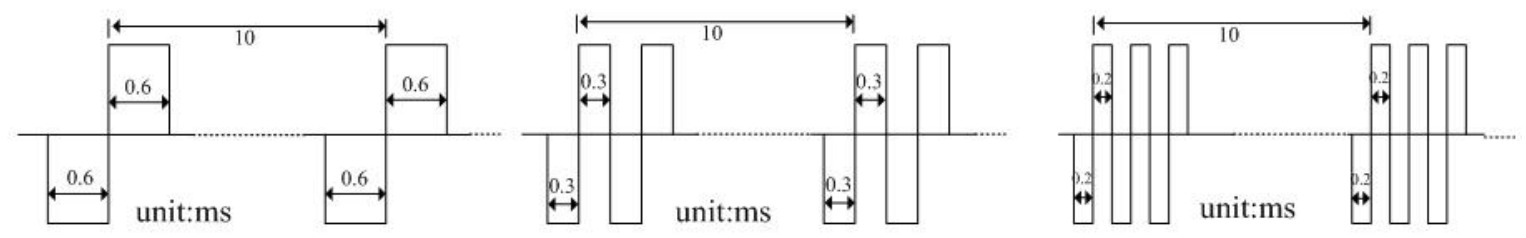

Fig. 2. Stimulation waveforms of three different modes with specified parameters (pulse number $=2$, duration $=0.6 \mathrm{~ms}$ and frequency $=100 \mathrm{~Hz}$.) (A) mode: 1 , pulse number $=2$, duration $=0.6 \mathrm{~ms}$ and frequency $=100 \mathrm{~Hz}$. (B) mode: 2 , pulse number $=2$, duration $=2 \times 0.3 \mathrm{~ms}$ and frequency $=100 \mathrm{~Hz}$. (C) mode: 3 , pulse number $=2$, duration $=3 \times 0.2 \mathrm{~ms}$ and frequency $=$ $100 \mathrm{~Hz}$. 
a microprocessor (C8051F006, Cygnal Inc.) and a single chip RF transceiver (nRF401, Nordic Inc.) powered by a $3.7 \mathrm{~V}, 120 \mathrm{mAh}$ polymer battery. All of the components were assembled on a printed circuit board, demonstrating a total current consumption of approximately $14 \mathrm{~mA}$. The receivermicroprocessor was mounted on the head of the robo-pigeon, as shown in Figure 1. It measured 26 $\mathrm{mm} \times 16 \mathrm{~mm} \times 9 \mathrm{~mm}$, weighed $9 \mathrm{~g}$, and could be easily carried by the bird.

Figure 3 shows the receiver-microprocessor circuitry in detail. Reg710-3.3 is a $3.3 \mathrm{~V}$ regulator. The programmable C8051F006 has 16 digital I/O pins: two of these pins were set to communicate with the $\mathrm{nRF} 401$ as a serial port, and three pins were used to output to the tricolor LED. Six pins were retained to operate two dual 4-channel multiplexers (DG4062A, Vishay Intertechnology, Inc.) aligned in series, as shown in Figure 3. One multiplexer was set to generate a biphasic pulse by setting three C8051F006 pins (pin number: $35,36,37$ ) to low or high voltage. Another multiplexer was used to set up three separate output channels by setting three C8051F006 pins (pin number: 38, 39, 40) to low or high voltage. Each output channel used two multiplexer pins (CHAN1_A and CHAN1_B, CHAN2_A and CHAN2_B, CHAN3_A and CHAN3_B) to stimulate its respective brain region with constant voltage biphasic stimulus pulses. Pulse amplitudes were approximately $3.7-4.0 \mathrm{~V}$, depending on the battery voltage. The remaining pins were available for channel expansion.

The microprocessor was pre-loaded with a $\mathrm{C}$ program to control stimulation; the program was designed to wait for an ASCII command from nRF401. As soon as a valid message was received, it branched to the setting routine or the stimulation routine according to the received identifier. During stimulation, the analog switches corresponding only to the requested channel were switched on; the other switches remained off to prevent interference between the electrodes.

The three paired output pins of the DG4062A were connected to a $2.54 \mathrm{~mm}$ single-row pin header plugged into a skull-top female connector which housed the electrodes. Stimulation pulses were outputted from the pin header to the brain regions via the electrodes. A tricolor LED was mounted on the printed circuit board to provide direct visual verification of the receiver-microprocessor state. The

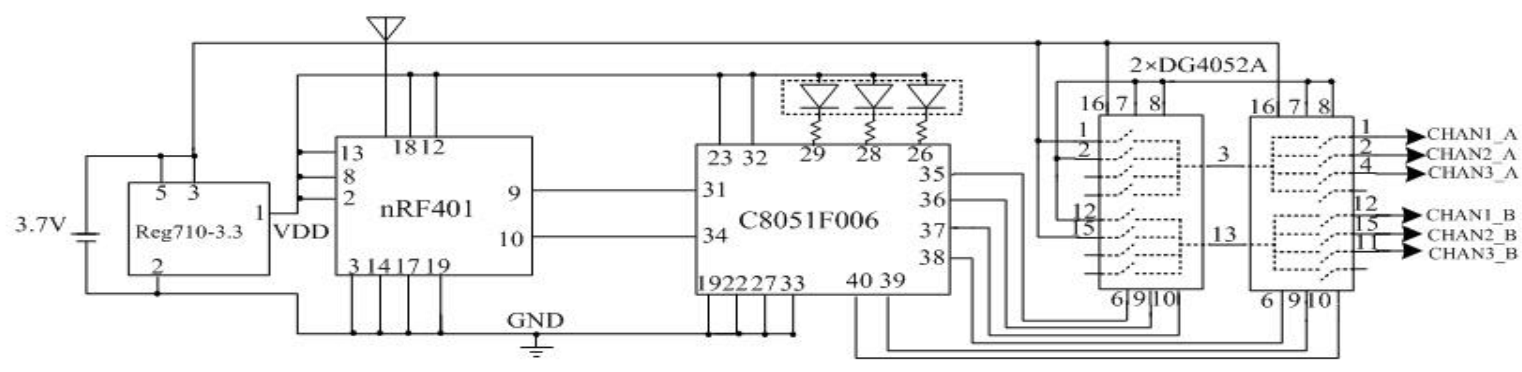

Fig. 3. Schematic diagram of the receiver-microprocessor.
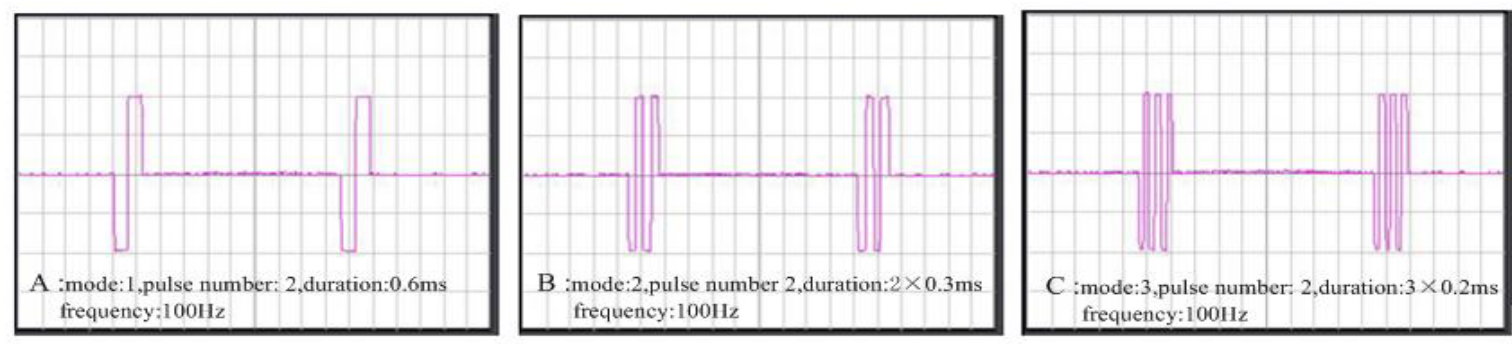

Fig. 4. Stimulation waveforms of the receiver-microprocessor. 
receiver-microprocessor outputted multi-mode stimuli by randomly alternating three pulse modes. Figure 4 illustrates the stimulation waveform output of the new receiver-microprocessor, acquired by oscilloscope (GDS-810C, GW Instek).

\section{Experimental results and discussion}

The effectiveness of the multi-mode telestimulation system was confirmed using a conventional robo-pigeon test primarily conducted in a cage. This test of robo-pigeon navigation was designed to demonstrate the feature of the multi-mode telestimulation system and clearly elucidate the virtual fear behavioral model. The objective of the experiment was to remotely guide the robo-pigeon to walk along a clearly marked route, under the control of the new multi-mode telestimulation system. Blue adhesive tape (length $5 \mathrm{~m}$, width $3 \mathrm{~cm}$ ) was set in a straight line on the ground in a large room, and served as the target route for the test. The receiver-microprocessor was mounted to the head of the pigeon via an adapter. To prevent the pigeon from flying, the wings of the pigeon were tied with tape.

The receiver-microprocessor displayed three independent output channels; it was possible to specify the parameters of each channel. The parameter ranges were as follows: the number of pulses was 615; the pulse duration was $0.4-0.8 \mathrm{~ms}$; and the pulse frequency was $80-120 \mathrm{~Hz}$. Before the navigation experiment, the initial default parameters (pulse number $=10$, pulse duration $=0.6 \mathrm{~ms}$, pulse frequency $=100 \mathrm{~Hz}$.) of the three channels were pre-programmed, and the test commenced with the multi-mode telestimulation system in a cage. The parameters were adjusted according to robopigeon's behavioral response to stimulation. When the experiment began, only the commands were transferred, and the parameters did not need to be transferred repeatedly. The commands contained a short header and the ID of the channel to be stimulated.

A series of pictures of the experimental process is shown in Figure 5, and the complete experimental results drawn from video recorded during the experiment are shown in Figure 6. When the robopigeon deviated from the target route, the operator sent a remote command to the receivermicroprocessor using the telemetry system to stimulate the right or left DIVA via the electrodes. Thus, the operator guided the robo-pigeon to turn toward the target route. Similarly, stimulation of the PAG made the robo-pigeon move forward from a stationary state. The robo-pigeon walked on the target route in a zigzag pattern from the start to the destination, under prolonged brain-stimulation. The total time of the experiment was 99 seconds. The dashed line represents the target route, the solid curve represents the actual walking route of the robo-pigeon under brain-stimulation, and the dot marked along the curve corresponds to each time label.

For single mode stimulation (pulse number $=10$, pulse duration $=0.6 \mathrm{~ms}$, pulse frequency $=100$ Hz.), the sensitivity of the robo-pigeon decreased after approximately one minute under prolonged steady stimulus [12]. For multi-mode stimulation, experimental results demonstrated that the robopigeon remained in a state of strong control during the entire experimental navigation process, under prolonged multi-mode and non-steady stimulus. In terms of a stimulation system, the effect of a stimulus pulse train is not only dependent upon pulse amplitude, pulse duration, pulse frequency and the total number of pulses, but is also dependent upon the pulse mode which has been previously identified as an important factor in robo-pigeon navigation.

In a situation similar to the test protocols, a robo-animal based on virtual fear could be utilized as a robot after surgical rehabilitation (approximately seven days). However, whether or not the roboanimal based on virtual reward could become a robot is heavily dependent on specialized training, which helps the animals to learn specific behaviors $[4,9]$. Therefore, the virtual fear behavioral model 

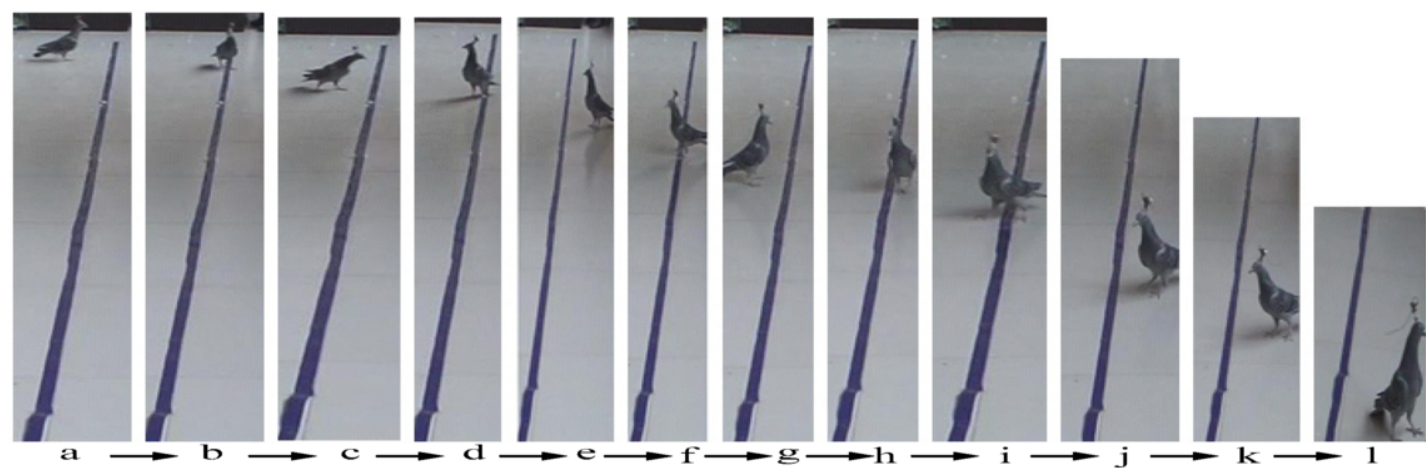

Fig. 5. Pictures of robo-pigeon navigation experiment derived from video evidence.

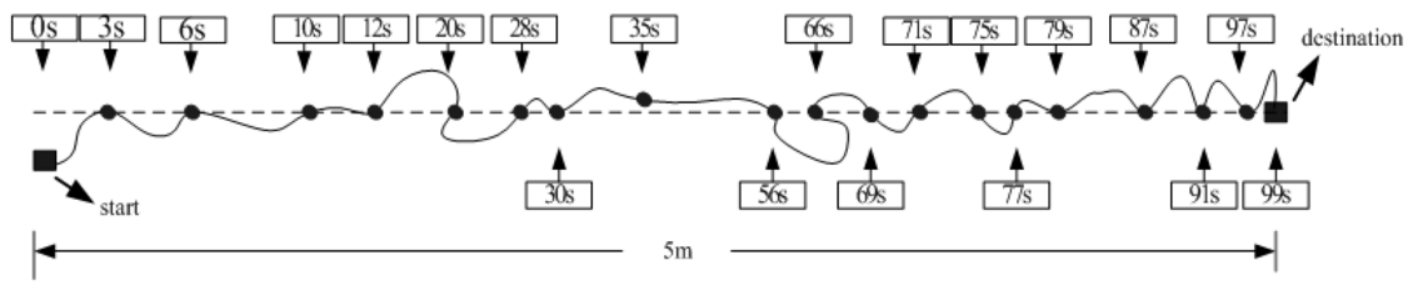

Fig. 6. Experimental results of robo-pigeon navigation under multi-mode brain microstimulation.

is faster and more efficient than a virtual reward model.

\section{Conclusion}

The multi-mode telestimulation system described here alleviates the adaptation of neurons, observed in the previously described single-mode stimulation system, by the provision of multi-mode and nonsteady TTL biphasic pulses. Furthermore, the proposed multi-mode telestimulation system is smaller and lighter than previous telemetry systems. In this study, a new animal behavioral model (virtual fear) was presented and demonstrated to be more practical and efficient than the previous behavioral model (virtual reward), because it does not require time-consuming and laborious training of the test animal. The multi-mode telestimulation system and the virtual fear behavioral model could be highly useful in the study of robo-animals, biological sensors and other bionic engineering or biomedical engineering applications. Although this system presents clear advantages, there remain some challenges. For example, the stimulator should be designed into an ASIC and fully implanted into the body of the animal to improve the reliability and practicability of the robo-animals.

\section{Acknowledgments}

This work was supported by the National Natural Science Foundation of China (Nos. 61203370, 61305129, 60875068), International Cooperation Projects of the Ministry of Science and Technology of China (NO. 2008DFR00560), Natural Science Foundation of Shandong Province (ZR2013CQ017) and Qingdao Economic and Technological Development Zone Program (\#2013-1-36). 


\section{References}

[1] R.R. Harrison, H. Fotowat, R. Chan, R.J. Kier, A. Leonardo and F. Gabbiani, A wireless neural/ EMG telemetry system for freely moving insects, Proceedings of the 2010 IEEE International Symposium on Circuits and Systems, Paris, France, 2010, 2940-2943.

[2] S.H. Xu, S.K. Talwar, E.S. Hawley, L. Li and J.K. Chapin, A multi-channel telemetry system for brain microstimulation in freely roaming animals, Journal of Neuroscience Methods 133 (2004), 57-63.

[3] W.G. Song, J. Chai, T.Z. Han and K. Yuan, A remote controlled multimode micro-stimulator for freely moving animals, Acta Physiologica Sinica 58 (2006), 183-189.

[4] W.M. Tsang, A.L. Stone, D. Otten, Z.N. Aldworth, T.L. Daniel, J.G. Hildebrand, R.B. Levine and J. Voldman, Insectmachine interface: A carbon nanotube-enhanced flexible neural probe, Journal of Neuroscience Methods 204 (2012), 355-365.

[5] W.M. Tsang, A.L. Stone, Z.N. Aldworth, J.G. Hildebrand, T.L. Daniel, A.I. Akinwande and J. Voldman, Flexible splitring electrode for insect flight biasing using multisite neural stimulation, IEEE Transactions on Biomedical Engineering 57 (2010), 1757-1764.

[6] C. Wang, T. Sung, Y. Wu and C. Hsu, A reconfigurable 16-channel HV stimulator ASIC for spinal cord stimulation systems, 2012 IEEE Asia Pacific Conference on Circuits and Systems, Kaohsiung, Taiwan, 2012, 300-303.

[7] J.Y.J. Tan, X. Liu, K.H. Wee, S.C. Yen, Y.P. Xu and T.C. Tan, A monolithic programmable nerve/muscle stimulator, 5th International IEEE/EMBS Conference on Neural Engineering, Cancun, Mexico, 2011, 511-514.

[8] R.Holzer and I. Shimoyama, Locomotion control of a bio-robotic system via electric stimulation, IEEE/RSJ International Conference on Intelligent Robots and Systems, Piscataway, NJ, USA, 1997, 1514-1519.

[9] S.K. Talwar, S. Xu, E.S. Hawley, S.A. Weiss, K. A. Moxon and J. K. Chapin, Rat navigation guided by remote control, Nature 417 (2002), 37-38.

[10] H. Sato, C.W. Berry, B.E. Casey, G. Lavella, Y. Yao and M.M. Maharbiz, A cyborg beetle: Insect flight control through an implantable, tetherless microsystem, 21st IEEE International Conference on Micro Electro Mechanical Systems, Tucson, USA, 2008, 164-167.

[11] H. Sato, C.W. Berry, Y. Peeri, E.B. Brendan, B.E. Casey and G. Lavella, Remote radio control of insect flight, Frontiers in Integrative Neuroscience 3 (2009), 1-11.

[12]X.C. Su, R.T. Huai, J.Q. Yang, H. Wang and C.Z. Lv, Brain mechanism and methods for robo-animal motor behavior control, Science China Information Sciences 9 (2012), 1130-1146.

[13] A. Bozkurt, R.F. Gilmour and A. Lal, Balloon-assisted flight of radio-controlled insect biobots, IEEE Transactions on Biomedical Engineering 56 (2009), 2304-2307.

[14] P.H. Peckham and J.S. Knutson, Functional electrical stimulation for neuromuscular applications, Annual Review of Biomedical Engineering 7 (2005), 327-360.

[15] W.B. Wang, Z.D. Dai, H. Tan, C. Guo and J.R. Sun, A stereotaxic method and apparatus for the Gekko gecko, Chinese Science Bulletin 53 (2008), 1107-1112.

[16]D. Zhang, Y.L. Dong, M.G. Li and H.J. Wang, A radio-telemetry system for navigation and recording neuronal activity in free-roaming rats, Journal of Bionic Engineering 9 (2012), 402-410.

[17]Z.Y. Feng, W.D. Chen, X.S. Ye, X.M. Zhang, X.J. Zheng, P. Wang, J. Jang, Z.J. Xu, C.Q. Liu, F.X. Liu, J.B. Luo, Y.T. Zhuang and X.X. Zheng, A remote control training system for rat navigation in complicated environment, Journal of Zhejiang University: Science A 8 (2007), 323-330.

[18] E.D. Adrian, The Mechanism of Nervous Action: Electrical Studies of the Neuron, University of Philadelphia Press, Philadelphia, 1932.

[19]P.J. Liang and A.H. Chen, Neuronal Activity Multi-Electrode Recorded Simultaneously and the Neural Information Processing, Beijing University of Technology Press, Beijing, 2003.

[20] S.A. Prescott and T.J. Sejnowski, Spike-rate coding and spike-time coding are affected oppositely by different adaptation mechanisms, Journal of Neuroscience 28 (2008), 13649-13661. 